\title{
DISTRIBUCIÓN DE FRACCIONES DE PROTEÍNA Y SU CONTRIBUCIÓN A LAS CARACTERÍSTICAS DE CALIDAD DE TRIGO
}

\section{PROTEIN FRACTION DISTRIBUTION AND THEIR CONTRIBUTION TO QUALITY CHARACTERISTICS IN WHEAT}

\author{
Nayelli Hernández Espinosa ${ }^{1}$, Gabriel Posadas Romanoํㅜ, Fausto Cervantes López ${ }^{1}$, Héctor I. González Santoyo ${ }^{1}$, \\ Amalio Santacruz Varela ${ }^{2}$ Ignacio Benítez Riquelme ${ }^{2}$ y Roberto J. Peña Bautista ${ }^{1 \star}$
}

${ }^{1}$ Centro Internacional de Mejoramiento de Maíz y Trigo. km 45 carr. México-Veracruz. El Batan. 56130,Texcoco, Edo. de México. Tel (595) 9521900 ext.1244 ó 2236. ${ }^{2}$ Campus Montecillo, Colegio de Postgraduados. km 36.5 Carretera México-Texcoco. 56230, Montecillo, Texcoco, Edo. de México.

${ }^{*}$ Autor para correspondencia (j.pena@cgiar.org)

\section{RESUMEN}

Las características visco-elásticas del gluten del trigo (Triticum aestivum $\mathrm{L}$.) están definidas por la composición de las gliadinas, las gluteninas de alto (G-APM) y bajo (G-BPM) peso molecular y por la distribución de sus principales fracciones, la monomérica (rica en gliadina, PM), la polimérica soluble (rica en glutenina soluble, PPS) y la polimérica insoluble (rica en glutenina insoluble, PPI). Con el objetivo de examinar la relación e influencia de estas fracciones sobre las características reológicas de calidad de procesamiento, se evaluaron 117 líneas de trigo procedentes de seis poblaciones contrastantes en calidad, cultivadas bajo riego en Sonora, México (ciclo 2005-2006). La variedad 'Avocet' fue el progenitor materno común en cada una de las seis poblaciones. Se consideraron dos variantes en la composición de gluteninas en los loci Glu-A1, Glu-B1, Glu-D1, y tres en el locus Glu-B3. Las medias generales de las proporciones de las tres fracciones fueron de $42 \%, 20.4 \%$ y $37.5 \%$ para PM, PPS y PPI, respectivamente. La distribución de fracciones fue distinta entre las seis poblaciones. El análisis estadístico mostró valores de predicción mayores a $30 \%$ de las fracciones proteicas y del contenido de proteína, para estimar variables de calidad del mixógrafo, de fuerza de masa, y de fuerza del gluten medida con el alveógrafo. La magnitud del efecto de las fracciones sobre las características de calidad varió entre las poblaciones de trigo evaluadas. La fracción PM mostró correlaciones significativas con características de extensibilidad de la masa (Sedim-SDS; AlvP/L), mientras que la fracción PPI correlacionó con las variables de fuerza de gluten (MIXTD, \%Tq/min y AlvW). La determinación de la distribución de fracciones proteicas, de la composición de G-APM y G-BPM, aunada a pruebas de elaboración rápida en laboratorio (proteína y Sedim-SDS), constituyen una importante herramienta para selección por calidad en el fitomejoramiento del trigo.

Palabras clave: Triticum aestivum, trigo harinero, proteínas monoméricas, proteínas poliméricas, propiedades visco-elásticas.

\section{SUMMARY}

Wheat visco-elastic properties (Triticum aestivum L.) are mainly defined by the composition of gliadins, high (HMW-G) and low (LMW-G) molecular weight glutenins, and by the distribution of main protein fractions, monomeric (MP, rich in gliadins), soluble polymeric (SPP, rich in soluble glutenin) and insoluble polymeric (IPP, rich in insoluble glutenin). The relationship and influence of these protein fractions on rheological characteristics was examined on a set of 117 lines derived from six populations of contrasting quality. Each line had 'Avocet' as common female parent, and all of them grew in Sonora,
México (2005-06 crop cycle). Two allelic variants in Glu-A1, Glu-B1, $G l u-D 1$, and three in Glu-B3 loci were considered in the analysis. Overall mean distribution of the protein fraction was $42 \%, 20.4 \%$, and $37.5 \%$ for MP, SPP, and IPP, respectively. However, the means for the three fractions varied among the six wheat populations. The statistical analysis showed predicting values above $30 \%$ between some protein fractions and protein content, for estimation of dough mixograph traits and alveograph trait $\mathrm{W}$. The effect of protein fractions distribution on wheat quality traits varied among the six genotypic populations examined. The MP showed significant correlation coefficients with dough extensibility values (Sedim-SDS and AlvP/L), while IPP correlated significantly with gluten strength data (MIXTD, \% Tq, and AlvW). Determination of protein solubility distribution, Glu-1/Glu-3 allelic combinations, combined with rapid small-scale tests (protein and SDS-Sedimentation), represent a powerful selection strategy in wheat breeding to identify sources of desirable quality traits for new crosses, and later on to screen desirable quality parameters.

Index words: Triticum aestivum, bread wheat, monomeric proteins, polymeric proteins, visco-elastic properties.

\section{INTRODUCCIÓN}

En trigo (Triticum aestivum L.) las proteínas de reserva comprenden de 80 a $85 \%$ del total de proteínas presentes en el grano (Pomeranz, 1978). Estas proteínas de reserva ya hidratadas y orientadas forman una red insoluble llamada gluten (Weegels, 1996). Las propiedades visco-elásticas del gluten dependen de factores como el genotipo (composición de gliadinas y gluteninas controlados por el complejo loci $G l i-G l u$ ) y la distribución de las principales fracciones de proteínas de gluten (gliadinas y gluteninas). Las gliadinas, también conocidas como proteínas monoméricas por sus agregados simples de polipéptidos, son proteínas de reserva presentes en el endospermo, se componen de las sub-unidades $\alpha, \beta, \gamma \mathrm{y} \omega$-gliadinas, y se caracterizan por su solubilidad en alcohol a diferentes concentraciones (Shewry et al., 2002). Estas fracciones de proteínas están asociadas con la viscosidad de las masas (Sapirstein y Fu, 1998; Uthayakumaran et al., 1999; Suchy et al., 2007).

Las gluteninas son proteínas poliméricas también presentes 
en el endospermo, y están formadas por dos principales grupos de cadenas polipeptídicas llamadas subunidades de alto (APM) y bajo peso molecular (BPM), las cuales se mantienen unidas por puentes disulfuro. Estas proteínas se relacionan con el tiempo de desarrollo de la masa en el mixógrafo, la estabilidad en el farinógrafo y con el volumen de pan (Gupta et al., 1992; Uthayakumaran et al., 1999; Suchy et al., 2007).

Se han desarrollado diversos protocolos que separan y cuantifican la concentración de las principales fracciones de proteína y ayudan a esclarecer su relación con parámetros específicos de calidad (Bean et al., 1998; Figueroa et $a l ., 2009)$. Destacan tres protocolos que logran una mejor separación con métodos más simples (solubilidad). Sapirstein y Fu (1998) lograron aislar dos fracciones, una rica en proteínas monoméricas y otra rica en proteínas poliméricas, estas últimas divididas en gluteninas solubles e insolubles en alcohol. DuPont et al. (2005) publicaron un método más específico que logra separar a las proteínas monoméricas en una fracción rica en gliadina y otra en albuminas/globulinas. Suchy et al. (2007) separaron gluteninas monoméricas y poliméricas, y demostraron que es posible cuantificar las proporciones de proteína por espectrofotometría.

El estudio de las proteínas presentes en el grano de trigo así como de las diferentes interacciones con ellas (por efecto de cruzamiento, del ambiente o de una combinación de alelos), contribuye parcialmente a definir las características visco-elásticas y la calidad de panificación del trigo. Son escasos los estudios realizados sobre el comportamiento de las variaciones alélicas dentro del genoma del trigo para las fracciones de proteína. De la $\mathrm{O}$ et al. (2010) reportaron que el genotipo y el ambiente tienen efecto sobre las fracciones de glutenina y gliadina, y su contribución parcial en las características de calidad en trigo.

Es necesario, por tanto, investigar otros factores que permitan entender mejor el comportamiento complejo de la calidad industrial del trigo. Por ello, el presente trabajo tuvo como objetivo determinar la composición de subunidades de glutenina y la distribución de las fracciones ricas en gliadina (proteínas monoméricas, PM), en glutenina soluble (proteína polimérica soluble, PPS) y en glutenina insoluble (proteína polimérica insoluble, PPI), para valorar la posible influencia de estas fracciones sobre parámetros específicos de calidad, en líneas recombinantes de trigo.

\section{MATERIALES Y MÉTODOS}

\section{Material vegetal}

Las seis poblaciones recombinantes de trigo evaluadas tuvieron a la variedad 'Avocet' como progenitor materno común, la cual fue cruzada con las líneas experimentales 'Attila, 'Pastor,' 'Amadina', 'Parula,' 'Tonichi' y 'Pavón'. La progenie de cada población se derivó por descendencia de una sola semilla hasta $\mathrm{F}_{6}$, cultivada bajo condiciones de riego en Cd. Obregón, Sonora, donde la última generación se cosechó en el ciclo otoño-invierno 2005-2006. Los materiales utilizados en el estudio fueron sembrados por duplicado, y cada unidad experimental consistió de un surco doble de $2 \mathrm{~m}$ con $0.75 \mathrm{~cm}$ de espacio en cada surco doble. Para el manejo agronómico se aplicó fungicida y nitrógeno sin limitaciones.

'Avocet' destaca por su resistencia a la roya amarilla causada por el hongo Puccinia striiformis; las seis líneas experimentales son variedades de alto potencial de rendimiento pero contrastantes en calidad, vigentes en el programa de fitomejoramiento del Centro Internacional de Mejoramiento de Maíz y Trigo (CIMMYT).

De estas cruzas resultaron 400 líneas, de las cuales sólo se evaluaron las 117 líneas que fueron contrastantes en composición alélica, con las siguientes variantes: loci $G l u-A 1(0$, $\left.2^{*}\right)$, Glu-B1 $(7+8,17+18)$, Glu-D1 $(2+12,5+10)$ y Glu-B3 (b, $h, g)$. Todas las líneas mostraron en común las gluteninas Glu-A3c y Glu-D3b. Esta selección de líneas respondió a la necesidad de controlar la disparidad genotípica propia de la población y de poder analizar el efecto de cada alelo sobre las fracciones proteínicas analizadas.

\section{Evaluación de la calidad y determinación alélica}

Los análisis se realizaron en el Laboratorio de Química y Calidad de Cereales del CIMMYT, por duplicado. Muestras de $500 \mathrm{~g}$ de grano, previamente acondicionadas a niveles de humedad entre 14 y $16 \%$, de acuerdo con la dureza de grano, fueron molidas en un molino Brabender Quadrumat Jr. ${ }^{\circledR}$ (C. W. Brabender OHG, Germany), para obtener harina refinada en la cual se midieron las variables de calidad.

La dureza (DURGRN) y la humedad del grano fueron estimados por espectroscopía de reflectancia en el espectro infrarrojo cercano (NIRS, por sus siglas en inglés; NIRSystems $6500^{\mathrm{TM}}$, FOSS Dinamarca), conforme al método oficial AACC 39-70A (AACC, 2000). El contenido de proteína en harina (PROTREF) se estimó con espectrofotometría NIRS en el equipo INFRATEC $® 1255$ (FOSS-TECATOR, Dinamarca). Para el volumen de sedimentación (SedimSDS) se usó $1 \mathrm{~g}$ de harina refinada, con la metodología descrita por Peña et al. (1990).

Las propiedades de desarrollo de masa se determinaron con el mixógrafo de Swanson ${ }^{\circledR}$ (National Mfg., USA) mediante el método oficial 54-40A (AACC, 2000), en $35 \mathrm{~g}$ de harina refinada. Del mixograma se obtuvieron los datos de: 
tiempo de desarrollo de la masa (MIXTD), y el torque (Tq) al tiempo de desarrollo expresado en $\% \mathrm{Tq} / \mathrm{min}$, que se define como el trabajo necesario para mezclar el agua con la harina y lograr una masa con desarrollo óptimo (Walker y Walker, 2001). Adicionalmente, se utilizó el equipo Mixolab® System de Chopin (Trippette \& Renaud, Francia) para determinar propiedades de desarrollo de la masa: tiempo de desarrollo (TD-Mxlb), estabilidad al amasado (EstMxlb), ambas relacionadas con la calidad de gluten. Se usó un protocolo basado en las recomendaciones del fabricante, pero con modificaciones en la velocidad de desarrollo de masa $(200 \mathrm{rpm})$ y en la temperatura de calentamiento $\left(6^{\circ}\right.$ $\mathrm{C} / \mathrm{min}$ ). Las propiedades de fuerza y extensibilidad de la masa se determinaron en el alveógrafo Chopin ${ }^{\circledR}$ (Trippette \& Renaud, Francia) con el método 54-30A (AACC, 2000), en una muestra de $60 \mathrm{~g}$ de harina refinada; así se determinó tanto la fuerza general del gluten $\left(\mathrm{W} \times 10^{-4} \mathrm{~J}\right)$ como la relación tenacidad/extensibilidad $(\mathrm{P} / \mathrm{L})$.

La determinación alélica de gluteninas de alto peso molecular (APM) y bajo peso molecular (BPM) se efectuó con base en la separación de subunidades en gel de acrilamida con dodecil sulfato de sodio (SDS-PAGE, por sus siglas en inglés), con el método descrito por Peña et al. (2004); para la nomenclatura de G-APM se usó la identificación de Payne y Lawrence (1983), y para G-BPM la de Jackson et al. (1996) y Branlard et al. (2001).

\section{Fraccionamiento y cuantificación de proteínas}

Las muestras se procesaron por duplicado mediante extracción secuencial (Figura 1). De cada muestra se utilizaron $10 \mathrm{mg}$ de harina refinada (14\% de humedad, base húmeda) pesados directamente en tubos eppendorf de $2 \mathrm{~mL}$. Las PM (proteínas monoméricas) fueron extraídas con 1.8
$\mathrm{mL}$ de una solución que combina $2.3 \% \mathrm{NaI} / 3.75 \%$ 2-propanol, después de centrifugar por 3 min a $16500 \mathrm{Xg}$ y 25 ${ }^{\circ} \mathrm{C}$. Para la extracción de PPS (proteína polimérica soluble) se tomó el precipitado del tubo anterior y se le adicionó 1.8 $\mathrm{mL}$ de una solución preparada con $2 \% \mathrm{SDS} / 0.75 \%$ Tris $/ 40$ $\%$ de 2-propanol, y la mezcla se centrifugó a $25{ }^{\circ} \mathrm{C}$ por $5 \mathrm{~min}$ a $16500 \mathrm{Xg}$. Por último, se extrajo la fracción de PPI (proteína polimérica insoluble) después de añadir $1.8 \mathrm{~mL}$ de $0.2 \%$ DTT (dithiothreitol) $/ 50 \%$ 2-propanol e incubar por $1 \mathrm{~h} 15 \min$ a $55^{\circ} \mathrm{C}$.

Las muestras fueron incubadas a temperatura, velocidad y tiempo controlado con un incubador Eppendorf Thermomixer® Comfort (Eppendorf-Netheler, Hamburgo, Alemania). Para cuantificar las concentraciones de proteínas, se colocaron $100 \mu \mathrm{L}$ del sobrenadante en microplacas de 384 pozos para UV (marca CORNING), y la lectura se hizo en espectrofotómetro (Microplate reader BioTek Epoch ${ }^{\mathrm{TM}}$ ) a absorbancia de $280 \mathrm{~nm}$ (Suchy et al., 2007), y las lecturas se recuperaron con el programa Gen $5^{\mathrm{TM}}$ (Microplate Data Collection \& Analysis Software).

\section{Análisis estadístico}

Se calcularon las medias generales y desviaciones estándar de las concentraciones de proteína extraída por familia. Se hizo también una comparación de medias con la prueba de Tukey $(\mathrm{P} \leq 0.05)$, con el programa estadístico SAS versión 9.0 (SAS Institute, 2002). Los datos fueron transformados de mg a \%. Se aplicaron modelos de regresiones simples y múltiples acumulativos para estimar el porcentaje de explicación de las fracciones de proteína extraídas sobre los parámetros de calidad, con el programa estadístico CropStat 7.2.3, programa libre bajo los términos del IRRI

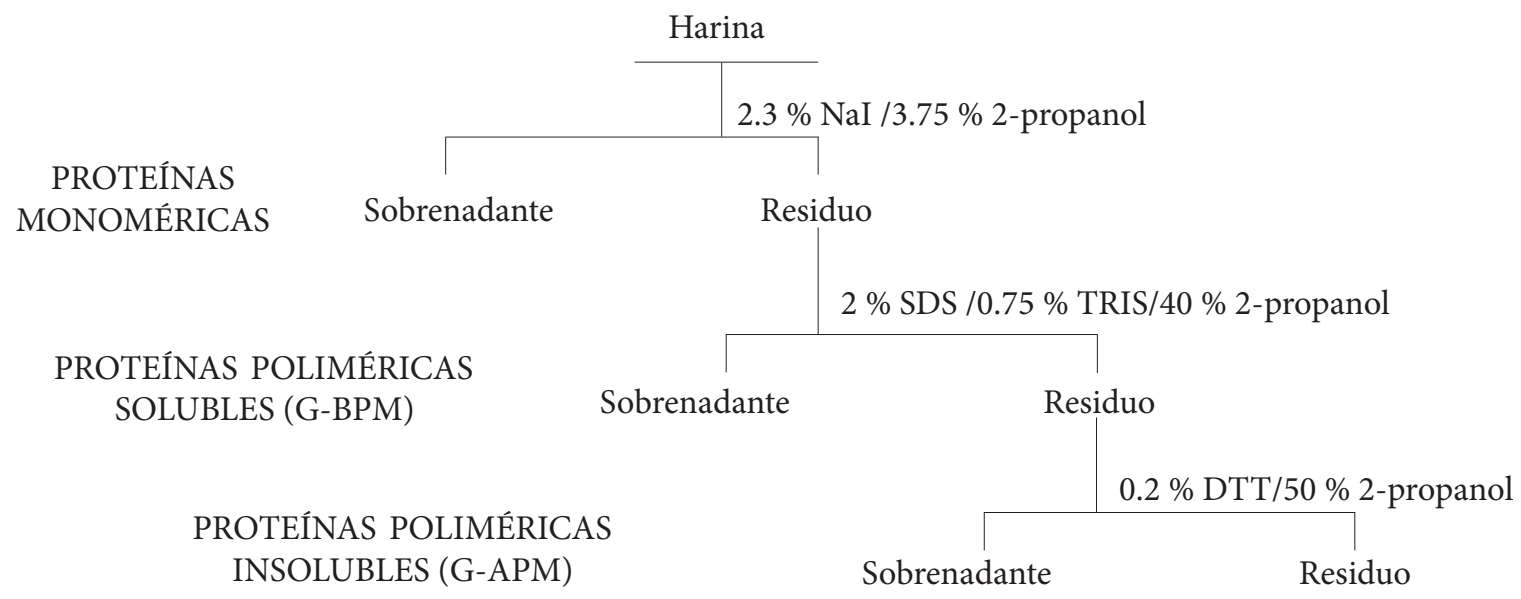

Figura 1. Diagrama de flujo de las etapas involucradas en el proceso de fraccionamiento de proteínas. 
(International Rice Research Institute). Mediante correlaciones de Pearson se determinó el grado de asociación entre las fracciones de proteína con las variables de calidad evaluadas, tanto de manera general como individual, de los alelos en los loci Glu-A1, Glu-B1, Glu-D1 y Glu-B3, y a nivel de combinaciones alélicas presentes en las poblaciones analizadas.

\section{RESULTADOS Y DISCUSIÓN}

En el Cuadro 1 se muestran valores mínimos, promedios y máximos encontrados en las líneas estudiadas para las características de calidad evaluadas, en donde se observa una amplia distribución de valores para todas las características, distribución asociada con las diferencias contrastantes en calidad de los progenitores involucrados en cada cruzamiento. La amplitud de valores se hace más evidente debido a que en la mayoría de las poblaciones se presentaron variantes alélicas para cada locus Glu-A1, Glu-B1, GluD1 y Glu-B3.

En el Cuadro 2 se presenta la distribución proporcional de las tres fracciones de proteína, desglosadas por cruza y en forma general. Se obtuvo una media general de $42 \%$ en proteína monomérica (PM), $20.4 \%$ en proteína polimérica soluble (PPS), y $39.1 \%$ en proteína polimérica insoluble (PPI). Estos resultados son similares a los valores de 50, 14 y $35.7 \%$ de PM, PPS y PPI, respectivamente, reportados por Sapirstein y Fu (1998) en trigos canadienses, y a los de DuPont et al. (2005) quienes reportaron $40.2 \%$ en la fracción rica en gliadina, 9.6 \% del complejo albúmina/globulina, y $48.0 \%$ de proteínas poliméricas, en líneas de trigos rojos de primavera.

\begin{tabular}{|c|c|c|c|}
\hline Variable & Mínimo & Promedio & Máximo \\
\hline DURGRN & 31 & 48 & 66 \\
\hline PROTREF (\%) & 8.0 & 10.0 & 13.2 \\
\hline Sedim-SDS (mL) & 7.5 & 10.0 & 13.8 \\
\hline MIXTD (min) & 1.1 & 2.3 & 4.4 \\
\hline$\% \mathrm{Tq} / \mathrm{min}$ & 42.5 & 94.9 & 186.8 \\
\hline TD-Mxlb (min) & 0.5 & 1.9 & 5.3 \\
\hline Est-Mxlb (min) & 1.1 & 2.7 & 5.7 \\
\hline AlvW $\left(10^{-4} \mathrm{~J}\right)$ & 55 & 240 & 494 \\
\hline AlvP/L & 0.6 & 1.6 & 4.5 \\
\hline \multicolumn{4}{|c|}{$\begin{array}{l}\text { DURGRN = dureza del grano; PROTREF = proteína en harina refinada; Sedim-SDS = se- } \\
\text { dimentación en harina; MIXTD = tiempo de desarrollo de la masa (en mixógrafo); \% Tq/ } \\
\text { min = torque al tiempo de desarrollo (en mixógrafo); TD-Mxlb = tiempo de desarrollo } \\
\text { (en mixolab); Est-Mxlb = estabilidad de la masa (en mixolab); AlvW = fuerza general del } \\
\text { gluten (en alveógrafo); AlvP/L = relación tenacidad/extensibilidad (en alveógrafo). }\end{array}$} \\
\hline
\end{tabular}

Cuadro 2. Comparación de medias de tratamientos respecto a las fracciones de proteínas extraídas en seis poblaciones de trigo.

\begin{tabular}{llll}
\hline Cruza & PM & \multicolumn{1}{c}{ PPS } & PPI \\
\hline 'Avocet' x 'Attila' & $40.4 \mathrm{ab}$ & $21.9 \mathrm{a}$ & $37.7 \mathrm{c}$ \\
'Avocet' x 'Pastor' & $39.5 \mathrm{~b}$ & $20.1 \mathrm{c}$ & $40.5 \mathrm{~b}$ \\
'Avocet' x 'Amadina' & $35.6 \mathrm{c}$ & $21.8 \mathrm{ab}$ & $42.6 \mathrm{a}$ \\
'Avocet' x 'Parula' & $39.9 \mathrm{~b}$ & $21.6 \mathrm{abc}$ & $38.4 \mathrm{c}$ \\
'Avocet' x 'Tonichi' & $41.0 \mathrm{ab}$ & $20.2 \mathrm{bc}$ & $38.8 \mathrm{bc}$ \\
'Avocet' x 'Pavón' & $42.1 \mathrm{a}$ & $20.4 \mathrm{abc}$ & $37.5 \mathrm{c}$ \\
Media general & $40.2 \%$ & $20.8 \%$ & $39.1 \%$ \\
\hline
\end{tabular}

Medias con la misma letra en cada columna, son estadísticamente iguales (Tukey, 0.05).PM = proteínas monoméricas; $\mathrm{PPS}$ = proteínas poliméricas solubles; $\mathrm{PPI}$ = proteínas poliméricas insolubles. 
Williams et al. (2008) y De la O et al. (2010) sugirieron que el genotipo y el ambiente son factores que afectan directamente a la expresión de la calidad. En las líneas provenientes de cruzas de una madre común con seis líneas experimentales contrastantes en calidad, se detectaron diferencias $(P \leq 0.05)$ en las proporciones de las fracciones extraídas; así, las cruzas de 'Avocet' con 'Pavón' y 'Tonichi' tuvieron la mayor proporción de $\mathrm{PM}$, mientras que las cruzas de 'Avocet' con 'Pastor' y 'Amadina' tuvieron la mayor proporción de PPI (Cuadro 2).

En un contexto general, las PM se relacionaron negativamente con las propiedades de fuerza de gluten y extensibilidad de la masa (Cuadro 3); ello indica que a mayor proporción de PM presentes, existiría menor fuerza de gluten y mayor extensibilidad en la masa. También Suchy et al. (2007) encontraron asociaciones negativas significativas de las PM contra seis características de calidad evaluadas en mixógrafo y cuatro en farinógrafo. Por su parte, $\mathrm{Hu}$ et al. (2007) reportaron relaciones positivas y significativas entre las PM y la extensibilidad, área de extensión y longitud de extensión, tres variables evaluadas en la elaboración de tallarines.

Las PPI presentaron relaciones positivas y significativas con características de fuerza de gluten en mixógrafo, mixolab y alveógrafo (Cuadro 3). Al respecto, Gupta et al. (1992) y Wang y Kovacs (2002) también obtuvieron relaciones significativas; los primeros autores con el conjunto de gluteninas (solubles e insolubles), y los segundos con la fracción
PPI, en ambos casos al estimar la asociación con medidas de fuerza evaluadas en mixógrafo, farinógrafo y alveógrafo. Además, Gupta et al. (1992) encontraron altas correlaciones con volumen de pan elaborado por métodos de fermentación de tiempo corto y tiempo largo.

Mediante análisis de regresión se estimó el porcentaje de explicación atribuible a las fracciones de proteína sobre características de calidad del trigo. La regresión simple mostró que las PM explican $14.0 \%$ del torque (\% Tq/min) y las PPI el $20.4 \%$ en volumen de sedimentación (Sedim-SDS) y 27.9 $\%$ de fuerza general de gluten (AlvW) (Cuadro 4). Estos resultados muestran una mayor influencia de las PPI que las PM, en la explicación y predicción de la calidad. Sin embargo, el análisis acumulativo destaca la acción combinada de las tres fracciones de proteína al explicar 30.2\%, $35.8 \%$ y $35.1 \%$ de tiempo de desarrollo de la masa (MIXTD), \% Tq/ min y AlvW, respectivamente; este tipo de análisis resulta muy útil en la elaboración de modelos matemáticos para la predicción de variables o parámetros reológicos de calidad.

En las líneas evaluadas se encontraron variaciones para los loci Glu-A1, Glu-B1, Glu-D1, Glu-B3 y sus alelos. La prueba de comparación de medias (Cuadro 5) permitió apreciar la relación de cada variante alélica con las fracciones de proteína extraída. Así, en el locus Glu-A1 la subunidad $2^{*}$ se asoció con mayor proporción de PM que el alelo nulo (0), mientras que este último se asoció con mayor contenido de PPI en comparación con el $2^{*}$. Luo et al. (2001) comparó el efecto de las gluteninas de bajo y alto peso molecular en características

Cuadro 3. Coeficientes de correlación de Pearson entre variables de calidad y proporciones de proteína en las líneas de trigo seleccionadas.

\begin{tabular}{llcc}
\hline Variable & PM & PPS & PPI \\
\hline DURGRN & 0.15 & -0.17 & -0.01 \\
PROTREF & -0.09 & 0.17 & -0.05 \\
Sedim-SDS & 0.03 & $-0.23^{*}$ & 0.16 \\
MIXTD & $-0.20^{*}$ & $-0.24^{*}$ & $0.41^{* *}$ \\
\%Tq/min & $-0.28^{* *}$ & -0.18 & $0.44^{* *}$ \\
TD-Mxlb & -0.14 & $-0.23^{*}$ & $0.33^{* *}$ \\
Est-Mxlb & -0.07 & $-0.45^{* *}$ & $0.44^{* *}$ \\
AlvW & $-0.31^{* *}$ & -0.12 & $0.41^{* *}$ \\
AlvP/L & $-0.21^{*}$ & 0.09 & 0.13 \\
\hline DURN
\end{tabular}

DURGRN = dureza del grano; PROTREF = proteína en harina refinada; Sedim-SDS = sedimentación en harina; MIXTD = tiempo de desarrollo de la masa (mixógrafo); $\% \mathrm{Tq} / \mathrm{min}=$ torque al tiempo de desarrollo (mixógrafo); TD-Mxlb = tiempo de desarrollo (mixolab); Est-Mxlb = estabilidad de la masa (mixolab); AlvW = fuerza general del gluten (alveógrafo); $\mathrm{AlvP} / \mathrm{L}=$ relación tenacidad/extensibilidad (alveógrafo); $\mathrm{PM}=$ proteínas monoméricas; $\mathrm{PPS}=$ proteínas poliméricas solubles; $\mathrm{PPI}=$ proteínas poliméricas insolubles. ${ }^{*} \mathrm{P} \leq 0.05,{ }^{* *} \mathrm{P} \leq 0.01$. 
de calidad de trigo, y encontró que la presencia de $2^{*}$ en el locus Glu-A1 se asocia con mayores volúmenes de pan que en presencia de la subunidad de glutenina nula $(0)$.

En el locus $G l u-B 1$ las subunidades $7+8,17+18$ no mostraron diferencia significativa en las medias de las proporciones de proteína extraída (Cuadro 5). El locus Glu-D1 arrojó valores similares entre alelos para la fracción de PM, mientras que la presencia de la subunidad $5+10$ mostró una proporción ligeramente mayor de PPI. Para el locus Glu-B3 se encontraron tres variantes alélicas, entre las que destaca el alelo $h$ con mayor proporción de PM, y el alelo $g$ con mayor proporción de PPI, aunque estadísticamente igual que el alelo $b$; en cambio, estas tres subunidades $h, b$, y $g$ presentaron diferencias significativas en contenidos medios de PPS.

Para el locus Glu-A1, De la O et al. (2010) no encontraron diferencias entre genotipos con los alelos $1,2^{\star}$ para la concentración de la fracción rica en gliadinas, pero la presencia de $2+12(G l u-D 1)$ se asoció con mayor cantidad de gliadinas; así mismo, para la fracción rica en glutenina las subunidades encontradas en el locus Glu-A1 no difirieron de manera significativa. Sin embargo, para el locus Glu-D1 la mayor cantidad de gluteninas correspondió a la presencia del alelo 5+10. En presencia de Glu-B3h se detectó un mayor contenido de la fracción rica en gliadina.

El análisis de correlación de Pearson del Cuadro 6 presenta la asociación entre las proporciones de proteína y las variables de calidad evaluadas, desglosada para cada variante alélica. En la fracción PM se encontró significancia negativa $(\mathrm{P} \leq 0.05$ o $\mathrm{P} \leq 0.01)$ para las correlaciones en las subunidades 0 , $2^{*}$ (Glu-A1); $7+8$ (Glu-B1); $2+12$, y $5+10$ (Glu-D1); b, g (Glu-B3). Estas correlaciones se presentaron en volumen de sedimentación y en variables evaluadas en mixógrafo, mixolab y alveógrafo, en función del efecto individual de cada alelo. En la fracción de PPI se encontraron

Cuadro 4. Análisis de regresión simple y múltiple de las fracciones extraídas de proteína contra características de calidad evaluadas en trigo.

\begin{tabular}{|c|c|c|c|c|}
\hline & \multicolumn{3}{|c|}{ Regresión simple } & \multirow{2}{*}{ Regresión múltiple } \\
\hline & PM & PPS & PPI & \\
\hline DURGRN & 0 & 3.1 & 0 & $\mathrm{PM}+\mathrm{PPS}=5.7$ \\
\hline PROTREF & 33.0 & 42.8 & 37.9 & $\mathrm{PM}+\mathrm{PPS}+\mathrm{PPI}=55.3$ \\
\hline Sedim-SDS & 11.2 & 0 & 20.4 & $\mathrm{PPI}=20.4$ \\
\hline MIXTD & 7.7 & 8.2 & 0 & $\mathrm{PM}+\mathrm{PPS}+\mathrm{PPI}=30.2$ \\
\hline$\% \mathrm{Tq} / \mathrm{min}$ & 14.0 & 4.6 & 5.8 & $\mathrm{PM}+\mathrm{PPS}+\mathrm{PPI}=35.8$ \\
\hline AlvW & 0 & 0 & 27.9 & $\mathrm{PM}+\mathrm{PPS}+\mathrm{PPI}=35.1$ \\
\hline AlvP/L & 0 & 0 & 0 & 0 \\
\hline
\end{tabular}

Cuadro 5. Comparación de medias por locus en las fracciones de proteínas extraídas en trigo.

\begin{tabular}{lcccc}
\hline \multirow{2}{*}{ Locus } & \multirow{3}{*}{ Subunidad/Alelo } & \multicolumn{3}{c}{ Fracciones de proteína (\%) } \\
\cline { 2 - 5 } Glu-A1 & 0 & $40.04 \mathrm{~b}$ & $20.69 \mathrm{a}$ & $39.25 \mathrm{a}$ \\
& $2^{*}$ & $41.28 \mathrm{a}$ & $21.02 \mathrm{a}$ & $37.69 \mathrm{~b}$ \\
Glu-B1 & $7+8$ & $40.08 \mathrm{a}$ & $20.79 \mathrm{a}$ & $39.11 \mathrm{a}$ \\
& $17+18$ & $40.50 \mathrm{a}$ & $20.71 \mathrm{a}$ & $38.77 \mathrm{a}$ \\
Glu-D1 & $2+12$ & $40.24 \mathrm{a}$ & $21.14 \mathrm{a}$ & $38.60 \mathrm{~b}$ \\
& $5+10$ & $40.15 \mathrm{a}$ & $20.32 \mathrm{~b}$ & $39.52 \mathrm{a}$ \\
Glu-B3 & $b$ & $39.60 \mathrm{~b}$ & $20.87 \mathrm{~b}$ & $39.52 \mathrm{a}$ \\
& $h$ & $41.60 \mathrm{a}$ & $21.37 \mathrm{a}$ & $37.01 \mathrm{~b}$ \\
& $g$ & $40.59 \mathrm{ab}$ & $19.06 \mathrm{c}$ & $40.33 \mathrm{a}$ \\
\hline
\end{tabular}

$\mathrm{PM}=$ proteínas monoméricas; PPS = proteínas poliméricas solubles; PPI = proteínas poliméricas insolubles. Medias con la misma letra dentro de columnas son estadísticamente iguales (Tukey, 0.05). 
correlaciones positivas con fuerza de gluten, en su mayoría altamente significativas $(\mathrm{P} \leq 0.01)$, como en el caso de las subunidades 0 (Glu-A1); 7+8 y $17+18$ (Glu-B1); $2+12$ y $5+10$ (Glu-D1); b y $g$ (Glu-B3). Su grado de influencia dependió del efecto de cada uno de los alelos.

Se obtuvieron ocho combinaciones alélicas de los loci Glu-A1, Glu-B1, Glu-D1 y Glu-B3 (Cuadro 7). Con referencia a PM destacó la combinación alélica 2 por tener correlaciones negativas y relevantes con variables de mixógrafo; de la misma forma, la combinación 3 correlacionó negativamente con AlvW, y la 7 con variables de Mixolab. Para las correlaciones encontradas con la PPI, las agrupaciones alélicas 1 y 7 presentaron mayor número de correlaciones significativas con variables de fuerza; en cambio, las combinaciones 2, 6 y 7 sobresalieron por presentar correlaciones significativas con la extensibilidad de la masa. Sólo la combinación 8 careció de correlación significativa entre las fracciones de proteína y las variables de calidad. Similarmente, De la O et al. (2010) concluyeron que para mejorar la cantidad de proteínas del gluten los genotipos idóneos deberían contener las combinaciones de alelos $2^{*} 17+18$ 5+10/ Glu-A3c, Glu-B3g, Glu-D3b y $117+185+10 /$ Glu-A3e, Glu-B3g, Glu-D3b.

La caracterización de proteínas de alto y bajo peso molecular, el fraccionamiento de las principales proteínas presentes en el grano de trigo, el análisis de sus combinaciones,

Cuadro 6. Correlaciones entre variables de calidad y las fracciones de proteínas extraídas, agrupadas por variantes alélicas.

\begin{tabular}{|c|c|c|c|c|c|c|c|c|c|c|}
\hline $\begin{array}{c}\text { Fracción } \\
(\%)\end{array}$ & Locus & Alelo & PROTREF & Sedim-SDS & MIXTD & $\% \mathrm{Tq} / \mathrm{mn}$ & TD-Mxlb & Est-Mxlb & AlvW & $\mathrm{AlvP} / \mathrm{L}$ \\
\hline \multirow[t]{2}{*}{$\mathrm{PM}$} & Glu-A1 & 0 & -0.14 & -0.10 & -0.17 & $-0.23^{*}$ & -0.18 & -0.07 & $-0.29^{* *}$ & $-0.20^{*}$ \\
\hline & & $2^{*}$ & -0.01 & $0.50^{\star \star}$ & 0.12 & 0.00 & -0.15 & -0.02 & -0.09 & -0.13 \\
\hline \multirow[t]{2}{*}{ PPS } & & 0 & 0.17 & $-0.24^{\star}$ & $-0.28^{\star \star}$ & $-0.24^{*}$ & $-0.20^{*}$ & $-0.46^{\star *}$ & -0.19 & 0.06 \\
\hline & & $2^{*}$ & 0.11 & -0.31 & -0.18 & -0.02 & $-0.38^{\star}$ & -0.30 & 0.09 & 0.30 \\
\hline \multirow[t]{2}{*}{ PPI } & & 0 & -0.01 & $0.31^{\star *}$ & $0.42^{* *}$ & $0.45^{\star *}$ & $0.36^{* *}$ & $0.48^{\star *}$ & $0.47^{* *}$ & 0.14 \\
\hline & & $2^{*}$ & -0.08 & -0.34 & 0.01 & 0.01 & 0.14 & 0.26 & 0.04 & -0.08 \\
\hline \multirow[t]{2}{*}{$\mathrm{PM}$} & Glu-B1 & $7+8$ & -0.19 & -0.11 & $-0.27^{\star}$ & $-0.42^{\star \star}$ & -0.20 & -0.08 & $-0.41^{\star \star}$ & -0.19 \\
\hline & & $17+18$ & 0.18 & 0.27 & -0.07 & 0.02 & -0.04 & -0.04 & -0.10 & -0.25 \\
\hline \multirow[t]{2}{*}{ PPS } & & $7+8$ & $0.25^{\star}$ & -0.20 & $-0.24^{\star}$ & -0.13 & $-0.24^{\star}$ & $-0.52^{\star \star}$ & -0.10 & 0.12 \\
\hline & & $17+18$ & -0.05 & -0.31 & -0.25 & -0.29 & -0.19 & -0.24 & -0.16 & 0.06 \\
\hline \multirow[t]{2}{*}{ PPI } & & $7+8$ & -0.02 & $0.26^{*}$ & $0.45^{\star *}$ & $0.51^{\star *}$ & $0.38^{\star *}$ & $0.49^{* *}$ & $0.47^{\star *}$ & 0.09 \\
\hline & & $17+18$ & -0.17 & -0.04 & $0.32^{\star}$ & 0.25 & 0.23 & 0.28 & 0.28 & 0.24 \\
\hline \multirow[t]{2}{*}{$\mathrm{PM}$} & Glu-D1 & $2+12$ & -0.12 & 0.02 & -0.24 & $-0.36^{* *}$ & $-0.26^{*}$ & -0.18 & -0.19 & -0.06 \\
\hline & & $5+10$ & -0.07 & 0.04 & -0.21 & $-0.28^{\star}$ & -0.03 & 0.04 & $-0.45^{\star \star}$ & $-0.34^{*}$ \\
\hline \multirow[t]{2}{*}{ PPS } & & $2+12$ & 0.13 & -0.19 & -0.18 & -0.08 & -0.05 & $-0.28^{\star}$ & 0.10 & 0.07 \\
\hline & & $5+10$ & 0.14 & -0.25 & -0.21 & -0.16 & $-0.33^{*}$ & $-0.58^{\star \star}$ & -0.03 & 0.22 \\
\hline \multirow[t]{2}{*}{ PPI } & & $2+12$ & 0.01 & 0.14 & $0.40^{* *}$ & $0.43^{* \star}$ & $0.31^{\star}$ & $0.42^{\star *}$ & $0.28^{\star}$ & 0.00 \\
\hline & & $5+10$ & -0.04 & 0.15 & $0.39^{* *}$ & $0.44^{* *}$ & $0.30^{*}$ & $0.43^{* *}$ & $0.51^{\star \star}$ & 0.19 \\
\hline \multirow[t]{3}{*}{$\mathrm{PM}$} & Glu-B3 & $b$ & -0.09 & -0.06 & -0.22 & $-0.29^{*}$ & $-0.25^{\star}$ & -0.08 & $-0.31^{\star *}$ & -0.07 \\
\hline & & $h$ & 0.06 & 0.12 & 0.11 & 0.03 & 0.18 & 0.15 & 0.08 & 0.03 \\
\hline & & $g$ & -0.36 & 0.40 & $-0.55^{\star}$ & $-0.57^{\star}$ & -0.19 & 0.08 & -0.36 & $-0.80^{\star *}$ \\
\hline \multirow[t]{3}{*}{ PPS } & & $b$ & $0.26^{*}$ & -0.07 & $0.26^{*}$ & -0.19 & -0.22 & $-0.47^{\star *}$ & 0.00 & 0.10 \\
\hline & & $\mathrm{h}$ & -0.18 & $-0.36^{*}$ & -0.21 & -0.13 & -0.33 & -0.30 & -0.30 & 0.01 \\
\hline & & $g$ & 0.33 & $-0.51^{\star}$ & 0.10 & 0.14 & 0.01 & -0.39 & -0.14 & 0.36 \\
\hline \multirow[t]{3}{*}{ PPI } & & $b$ & -0.10 & 0.12 & $0.43^{* *}$ & $0.45^{\star *}$ & $0.43^{* *}$ & $0.45^{\star *}$ & $0.32^{* *}$ & -0.01 \\
\hline & & $h$ & 0.11 & 0.21 & 0.08 & 0.10 & 0.11 & 0.11 & 0.20 & -0.05 \\
\hline & & $g$ & -0.05 & 0.25 & $0.49^{*}$ & 0.45 & 0.21 & 0.45 & $0.59^{*}$ & 0.40 \\
\hline
\end{tabular}

PROTREF = proteína en harina refinada; Sedim-SDS = sedimentación en harina; MIXTD = tiempo de desarrollo de la masa $(\mathrm{mixógrafo}) ; \% \mathrm{Tq} / \mathrm{min}=$ torque al tiempo de desarrollo (mixógrafo); TD-Mxlb = tiempo de desarrollo (mixolab); Est-Mxlb = estabilidad de la masa (mixolab); AlvW = fuerza general del gluten (alveógrafo); AlvP/L = relación tenacidad/extensibilidad (alveógrafo); $\mathrm{PM}$ = proteínas monoméricas; $\mathrm{PPS}$ = proteínas poliméricas solubles; $\mathrm{PPI}=$ proteínas poliméricas insolubles. $\mathrm{P} \leq 0.05,{ }^{*} \mathrm{P} \leq 0.01$. 


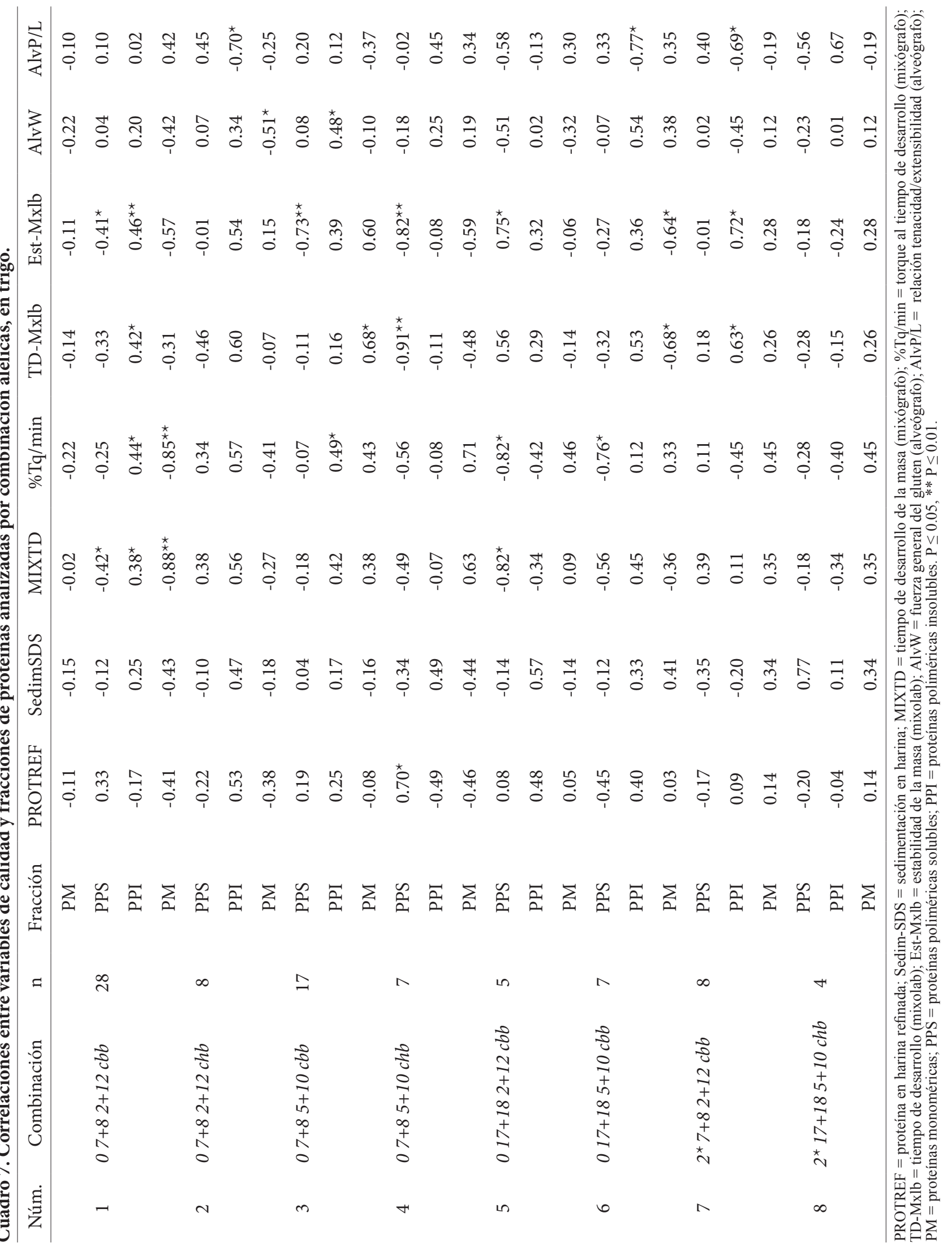


el efecto de éstas dentro de la cruza y su efecto sobre características importantes de la calidad, constituye una importante herramienta de apoyo al fitomejoramiento, porque permite realizar los cruzamientos entre progenitores sobresalientes y luego en su descendencia efectuar una selección más eficiente de líneas con los atributos de calidad que satisfagan las demandas específicas de la industria harinera.

\section{CONCLUSIONES}

En promedio de 117 líneas, los contenidos de fracciones de proteína fueron: $42 \%$ de monoméricas, $20.4 \%$ de poliméricas solubles y $37.5 \%$ de poliméricas insolubles, pero los contenidos variaron entre líneas de acuerdo con la cruza o la variación alélica particular del genotipo. El análisis de regresión demostró que la determinación de la distribución de fracciones de proteína constituye una herramienta complementaria en fitomejoramiento que en conjunto determinan en más de $30 \%$ el comportamiento de características importantes (tiempo de desarrollo de la masa, torque y fuerza general del gluten) de la calidad potencial de uso del trigo.

La proporción de las principales fracciones de proteína presentes en el trigo y su influencia sobre características importantes de calidad, es afectada por el genotipo tanto a nivel de variación alélica individual como en combinación de diferentes alelos.

Se confirma la influencia de las proteínas monoméricas en la extensibilidad de la masa (> PM, $>$ extensibilidad) y de las PPI sobre características de fuerza de gluten (> PPI, $>$ fuerza).

\section{AGRADECIMIENTOS}

Al financiamiento parcial del Fondo Sectorial SAGARPACONACYT-COFUPRO, Proyecto: 146788. Al equipo del Laboratorio de Química y Calidad de Trigo de CIMMYT, y a la colaboración conjunta de investigadores de CIMMYTColegio de Postgraduados.

\section{BIBLIOGRAFÍA}

AACC (2000) Approved Methods of the AACC. 10th ed. American Association of Cereal Chemists. St. Paul, MN, USA. 1268 p.

Bean S R, R K Lyne, K A Tilley, O K Chung, G L Lookhart (1998) A rapid method for quantitation of insoluble polymeric proteins in flour. Cereal Chem. 75:374-379.

Branlard G, M Dardevet, R Saccomano, F Lagoutte, J Gourdon (2001) Genetic diversity of wheat storage proteins and bread wheat quality. Euphytica 119:59-67.

De la O O M, E Espitia R, H E Villaseñor M, J D Molina G, H López S, A Santacruz V, R J Peña B (2010) Proteínas del gluten y reología de trigos harineros mexicanos influenciados por factores ambientales y genotípicos. Pesq. Agropec. Bras. 45:989-996.

DuPont F M, R Chan, R López, W H Vensel (2005) Sequential extraction and quantitative recovery of gliadins, glutenins, and other proteins from small samples of wheat flour. J. Agric. Food Chem. 53:1575-1584.

Figueroa J D C, T Maucher, W Reule, R J Peña (2009) Influence of high molecular weight glutenins on viscoelastic properties of intact wheat kernel and relation to functional properties of wheat dough. Cereal Chem. 86:139-144.

Gupta R B, I L Batey, F MacRitchie (1992) Relationships between protein composition and functional properties of wheat flours. Cereal Chem. 69:125-131.

Hu X Z, Y M Wei, C Wang, M I P Kovacs (2007) Quantitative assessment of protein fractions of Chinese wheat flours and their contribution to white salted noodle quality. Food Res. Int. 40:1-6.

International Rice Research Institute (IRRI). Crop Stat 7.2.3. Programa estadístico desarrollado para el análisis de datos en ensayos agrícolas. Disponible en: http://www.softpedia.es/programaCropStat-197357.html

Jackson E A, M H Morel, T Sontag-Strohm, G Branlard, E V Metakovsky, R Redaelli (1996) Proposal for combining the classification systems of alleles of Gli-1 and Glu-3 loci in bread wheat (Triticum aestivum L.). J. Genet. Breed. 50:321-336.

Luo C, W B Griffin, G Branlard, D L McNeil (2001) Comparison of lowand high molecular- weight wheat glutenin allele effects on flour quality. Theor. Appl. Genet. 102:1088-1098.

Payne P I, G J Lawrence (1983) Catalogue of alleles for the complex loci, Glu-A1, Glu-B1, and Glu-D1 which code for high-molecular-weight subunits of glutenin in hexaploid wheat. Cer. Res. Comm. 11:29-35.

Peña R J, A Amaya, S Rajaram, A Mujeeb-Kazi (1990) Variation in quality characteristics associated with some spring $1 \mathrm{~B} / 1 \mathrm{R}$ translocation wheats. J. Cereal Sci. 12:105-112.

Peña R J, H González-Santoyo, F Cervantes (2004) Relationship between $G l u-D 1 / G l u-B 3$ allelic combinations and bread-making quality-related parameters commonly used in wheat breeding. In: The Gluten Proteins. D Lafiandra, S Masci, R D’Ovidio (eds). Special Publication No. 295. Royal Society of Cheminstry. Cambridge, U.K. pp:156-157.

Pomeranz Y (1978) Wheat. Chemistry and Technology. American Association of Cereal Chemists Inc. St. Paul, Minnesota, USA. 821 p.

Sapirstein H D, B X Fu (1998) Intercultivar variation in the quantity of monomeric proteins, soluble and insoluble glutenin, and residue protein in wheat flour and relationships to breadmaking quality. Cereal Chem. 75:500-507.

SAS Institute (2002) SAS/STAT User's Guide, Software version 9.0. Cary, N. C, USA. 4424 p.

Shewry P R, N G Halford (2002) Cereal seed storage proteins: structures, properties and role in grain utilization. J. Exp. Bot. 53:947-958

Suchy J, O M Lukow, D Brown, R DePauw, S Fox, G Humphreys (2007) Rapid assessment of glutenin and gliadin in wheat by UV spectrophotometer. Crop Sci. 47:91-99.

Uthayakumaran S, P W Gras, F L Stoddard, F Bekes (1999) Effect of varying protein content and glutenin-to-gliadin ratio on the functional properties of wheat dough. Cereal Chem. 76:389394.

Walker A E, C E Walker (2001) Documentation and user's instructions for mixsmart version 4.0. National Cereal Chemistry Equipment. Lincoln, Nebraska, U.S.A. 150 p.

Wang C, M I P Kovacs (2002) Swelling index of glutenin test. II. Application in prediction of dough properties and end-use quality. Cereal Chem. 79:190-196.

Weegels P L, R J Hamer, J D Schofield (1996) Critical review: functional properties of wheat glutenin. J. Cereal Sci. 23:1-17.

Williams R M, L O’Brien, H A Eagles, V A Solah, V Jayasena (2008) The influences of genotype, environment, and genotype $\times$ environment interaction on wheat quality. Aust. J. Agric. Res. 59:95-111. 